\title{
The Absolute Magnitudes of Red Horizontal Branch Stars in the ugriz System
}

\author{
Y.Q. Chen, G. Zhao and J.K. Zhao
}

\begin{abstract}
Based on photometric data of the central parts of eight globular clusters and one open cluster presented by An and his collaborators, we select red horizontal branch $(\mathrm{RHB})$ stars in the $(g-r)_{0^{-}} g_{0}$ diagram and make a statistical study of the distributions of their colors and absolute magnitudes in the SDSS ugriz system. Meanwhile, absolute magnitudes in the Johnson VRI system are calculated through the translation formulae between gri and $V R I$ in the literature. The calibrations of absolute magnitude as functions of metallicity and age are established by linear regressions of the data.

It is found that metallicity coefficients in these calibrations decrease, while age coefficients increase, from the blue $u$ filter to the red $z$ filter. The calibration of $M_{\mathrm{i}}=0.06[\mathrm{Fe} / \mathrm{H}]+0.040 t+0.03$ has the smallest scatter of $0.04 \mathrm{mag}$, and thus $i$ is the best filter in the ugriz system when RHB stars are used for distance indicators. The comparison of the $M_{\text {I }}$ calibration from our data with that from red clump stars indicates that the previous suggestion that the $I$ filter is better than the $V$ filter in distance determination may not be true because of its significant dependence on age.
\end{abstract}

Subject headings: distance scale - globular clusters: general - stars: distances stars: horizontal-branch

\section{Introduction}

Stars with constant absolute magnitude are of high interest in astrophysics because they can be used as distance indicators. For a long time, horizontal branch stars have been widely adopted to determine distances of globular clusters and nearby galaxies. At present, various calibrations of absolute magnitude with metallicity are established by many works. For the

National Astronomical Observatories, Chinese Academy of Sciences, Beijing, 100012, China; gzhao@bao.ac.cn. 
low metallicity range of $[\mathrm{Fe} / \mathrm{H}] \leq-1.5$, absolute magnitudes in the $V$ band of RR Lyrae stars in globular clusters and in the field are investigated by using different methods and from different data. The results are not always consistent as shown in the review paper by Sandage $\&$ Tammann (2006). The coefficient $a$ in the linear relation of $M_{\mathrm{V}}=a[\mathrm{Fe} / \mathrm{H}]+c$ varies from 0.18 (Carretta et al. 2000) to 0.37 (Feast 1997) and the constant $c$ varies from 0.74 to 1.13 mag. In the solar neighborhood, absolute magnitudes in the $I$ band of red clump (hereafter $\mathrm{RC})$ stars in the metallicity range of $[\mathrm{Fe} / \mathrm{H}] \geq-0.5$ are well studied after the release of HIPPARCOS parallaxes (e.g. Stanek \& Garnavich 1998; Zhao et al. 2001; Groenewegen 2008). Most calibrations are consistent with a relation of $M_{\mathrm{I}}=0.13[\mathrm{Fe} / \mathrm{H}]-0.23$, and it is suggested that calibrations of absolute magnitude in the $I$ band are better than those in the $V$ band because they have a small dependence on metallicity. But, these calibrations do not take into account age dependence, probably due to the difficulty of deriving ages for $\mathrm{RC}$ stars. In view of this situation, it is interesting to investigate if the calibrations established from $\mathrm{RR}$ Lyrae stars with $[\mathrm{Fe} / \mathrm{H}]<-1.5$ are valid in the mild-metallicity range of $-1.5 \leq[\mathrm{Fe} / \mathrm{H}] \leq-0.5$. Moreover, one may ask how the calibrations of RR Lyrae and $\mathrm{RC}$ stars are connected at a fixed metallicity in between. In this respect, RHB stars may be important for establishing the calibrations for the mild metallicity population because they are significantly populated in the color-magnitude diagrams (hereafter CMDs) of mild metal poor globular clusters.

In this work, we aim to determine absolute magnitudes for RHB stars based on the photometric data of 17 globular clusters and three open clusters in the ugriz system presented in An et al. (2008). The main goals of this study are as follows. First, the calibrations of absolute magnitude can be established in the ugriz system and they are useful in distance determinations of interesting populations based on the large database of the SDSS photometric survey. Moreover, wavelength coverage of ugriz filters are narrower than those of Johnson $V$ and $I$ filters, and thus they provide more accurate magnitudes of RHB stars. Second, it is possible to investigate which filter, among the five ugriz filters, is the best distance indicator and how the absolute magnitude varies with metallicity and age. Finally, it is interesting to compare these calibrations and decide which calibration, in which filter, is the best for a particular population. Should we use different calibrations for a different metallicity range? Will the nonlinear calibrations with metallicity improve the precision of absolute magnitude determination? Is it necessary to include the age term in the calibrations? How are these calibrations adopted in different cases? 


\section{The selection of RHB stars}

The photometric data of 17 Galactic globular clusters and three open clusters in the SDSS ugriz system are taken from An et al. (2008) who applied the DAOPHOT/ALLFRAME programs to the central parts of clusters. These programs can provide more reliable ugriz photometric data for crowded cluster fields than those of the standard SDSS pipeline.

The selection of RHB stars in each cluster is based on the following procedures. As described in An et al. (2008), some clusters are observed more than one time and there are some overlapping regions between different observation runs. In view of this, the first step is to identify common stars in different observation runs and to provide a clear sample so that the same star observed for more than one time will not be considered as two or more stars. In the present work, stars with the same positions, i.e. both $\Delta|r a \cos (\operatorname{dec})|$ and $\Delta d e c$ being less than 0.5 arcsec, and the same colors, i.e. $\Delta g$ being less than 0.2 mag, in different observation runs are considered to be the same object and we include only one of them in the final sample. Then, we select stars within the tidal radius of the cluster and plot the CMD in different filters based on the reddening taken from Harris (1996), 2003 February version, which is presented in Table 1 of An et al. (2008). In the whole paper, the colors and magnitudes that we presented are reddening-corrected parameters.

Among the 20 clusters, eight globular clusters and one open cluster (presented in Table 1) show clear clumping of RHB stars. The nine clusters cover the metallicity range of $-1.7<[\mathrm{Fe} / \mathrm{H}]<+0.4$ and the age range of $8-12$ Gyr. The ages of the clusters are taken from Salaris \& Weiss (2002). As shown in Figure 1, the ages of five clusters increase with decreasing metallicity (they are classified as group 1) while four clusters (Pal 3,4,5,14) are significantly younger for their metallicity at $[\mathrm{Fe} / \mathrm{H}] \sim-1.5$ (they are classified as group 2 ). It is emphasized that clusters in group 2 are important, which makes it possible to investigate the age effect on the absolute magnitude in the present work. The two groups of clusters will be considered separately in the following analysis when necessary.

Usually, RHB stars in the CMD of each cluster, shown in Figure 2, are well separated from BHB stars, due to the presence of a gap in between, and from RGB stars, due to their low metallicity and/or old age. But we should keep in mind that the so-called RHB stars in this paper may include some RR Lyrae stars and even redder RC stars in clusters because we do not have suitable data or criteria to exclude them. Fortunately, it is expected that they will not affect the result of this paper in regards to statistics due to the small number and similar magnitudes in the HB of clusters.

The distributions of RHB stars are slightly different in the CMDs based on different colors and filters in the ugriz system. As shown in Figure 2, the $(g-r)_{0^{-}} g_{0}$ diagram of M5 


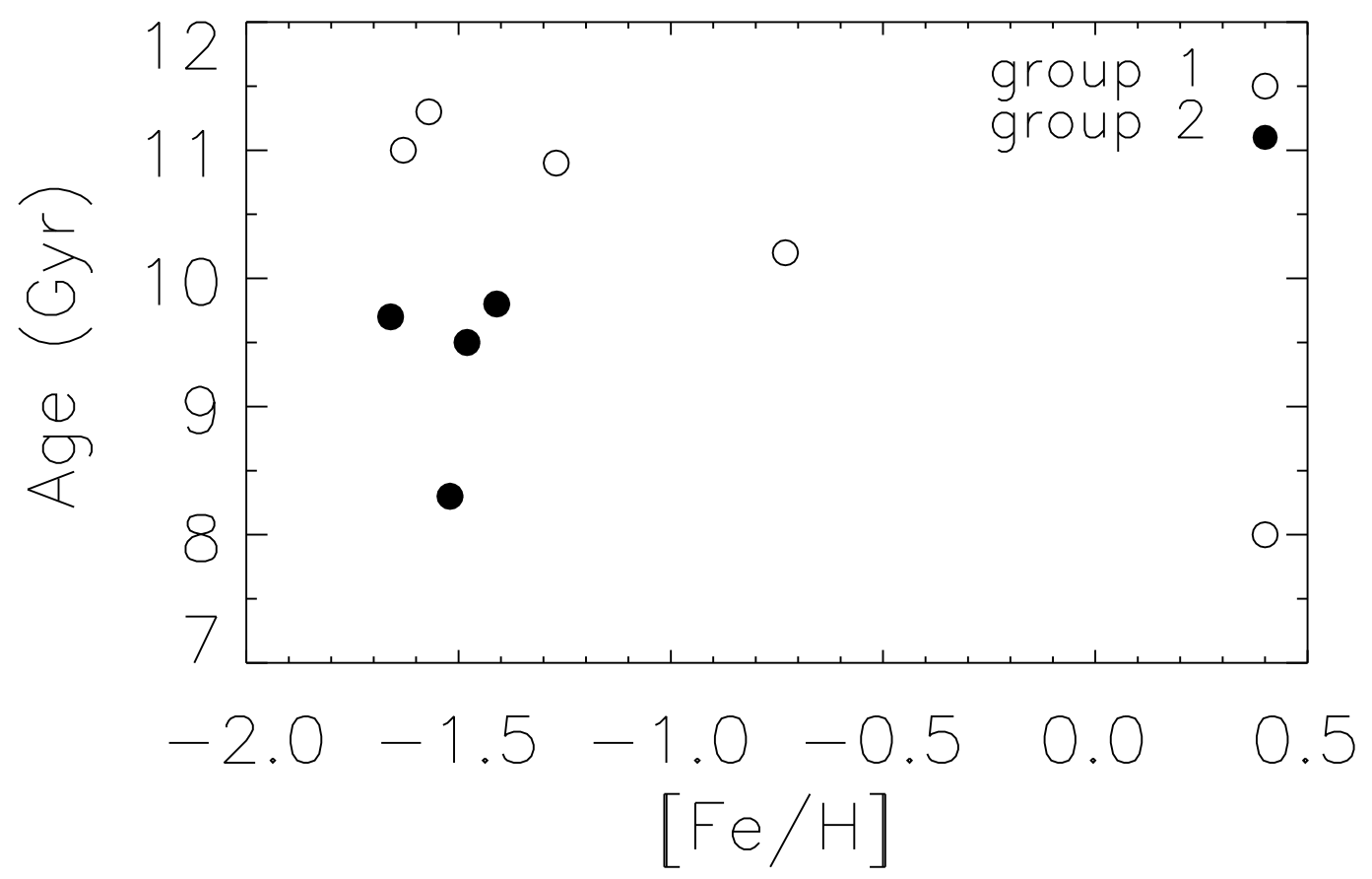

Fig. 1.- Age-metallicity diagram for nine clusters in group 1 (open) and group 2 (filled). 
is the best diagram to pick out RHB stars (within the defined box) in the sense that the gap between the BHB and RHB populations is clear and the RHB population is quite flat as $(g-r)_{0}$ varies. The RHB population is also clear in the $(g-r)_{0}-r_{0}$ diagram. However, it is difficult to pick out RHB stars based on the $(u-g)_{0}-u_{0}$ and $(u-g)_{0}-g_{0}$ diagrams because $(u-g)_{0}$ is a significantly metallicity-sensitive index, and RHB and BHB populations have the overlapping $(u-g)_{0}$ colors at 1.0-1.2 mag. Also, it is not easy to define the RHB population from the $(r-i)_{0}-i_{0}$ and the $(i-z)_{0}-z_{0}$ diagrams because the gaps between RHB and BHB/RGB are not so clear. Moreover, the photometric precisions of $g_{0}$ and $r_{0}$ filters are generally higher than those of $u_{0}, i_{0}$ and $z_{0}$ filters in the SDSS survey. Therefore, the $(g-r)_{0}-g_{0}$ diagram has the advantage of picking out RHB stars from these clusters.

In order to define the colors and magnitude ranges of RHB stars in the $(g-r)_{0^{-}} g_{0}$ diagram, we adopt a critical radius ( $R s$ in Table 1 ) for each cluster, within which stars are selected to be plotted in the CMD. The exception is Pal 4, for which we select all stars presented in the An et al. (2008) paper without using any critical radius because there are not enough stars even within the tidal radius. The half diameter from van den Bergh (2006) is adopted to be the critical radius for open cluster NGC 6791. For the remaining clusters, a critical radius between half light radius and tidal radius is chosen so that the RHB population in the CMD will become more clear. Then, we can define colors and magnitude ranges of RHB stars for clusters by eye. As we show later, the resulting absolute magnitudes will not be affected by the exact edges of the defined colors and magnitudes of RHB populations. Figure 3 shows the defined boxes of RHB populations based on the $(g-r)_{0} r_{0}$ diagrams for the other eight clusters except for M5 which is already shown in Figure 2.

\section{Results and Discussions}

\subsection{The color and absolute magnitudes of RHB stars}

With the defined colors and magnitude ranges of RHB stars in Figures 2 and 3, we select all possible RHB candidates. Then, Gaussian fittings to the color and magnitude distributions of the selected RHB stars provide the centering values and their scatters. As an example, Figure 4 shows the Gaussian fits to the distributions of $(g-r)_{0}, g_{0}, u_{0}, r_{0}$, $i_{0}$, and $z_{0}$ for $\mathrm{M} 5$ at $[\mathrm{Fe} / \mathrm{H}]=-1.27$. Usually, these distributions have sharp peaks and do not exactly obey Gaussian functions. Thus, the scatters from these Gaussian fits may be meaningless, but the centering values are generally consistent with these sharp peaks. Meanwhile, it is clear that the distributions of $z_{0}$ magnitudes are broader than those of ugri magnitudes. We note that photometric errors in the SDSS survey cannot explain this

difference because the errors in ugriz are about 0.01-0.02 mag for RHB stars in M5 with 

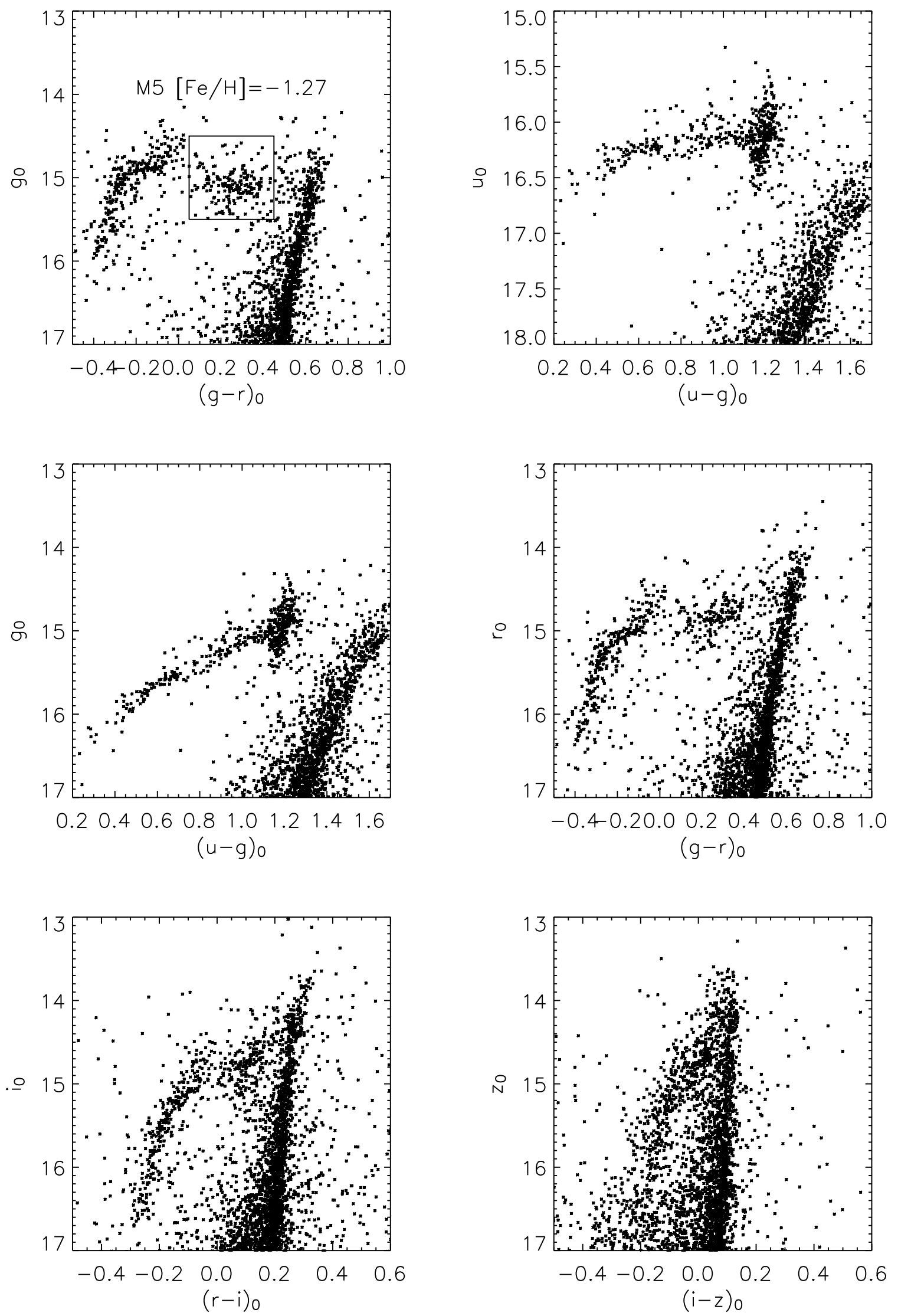

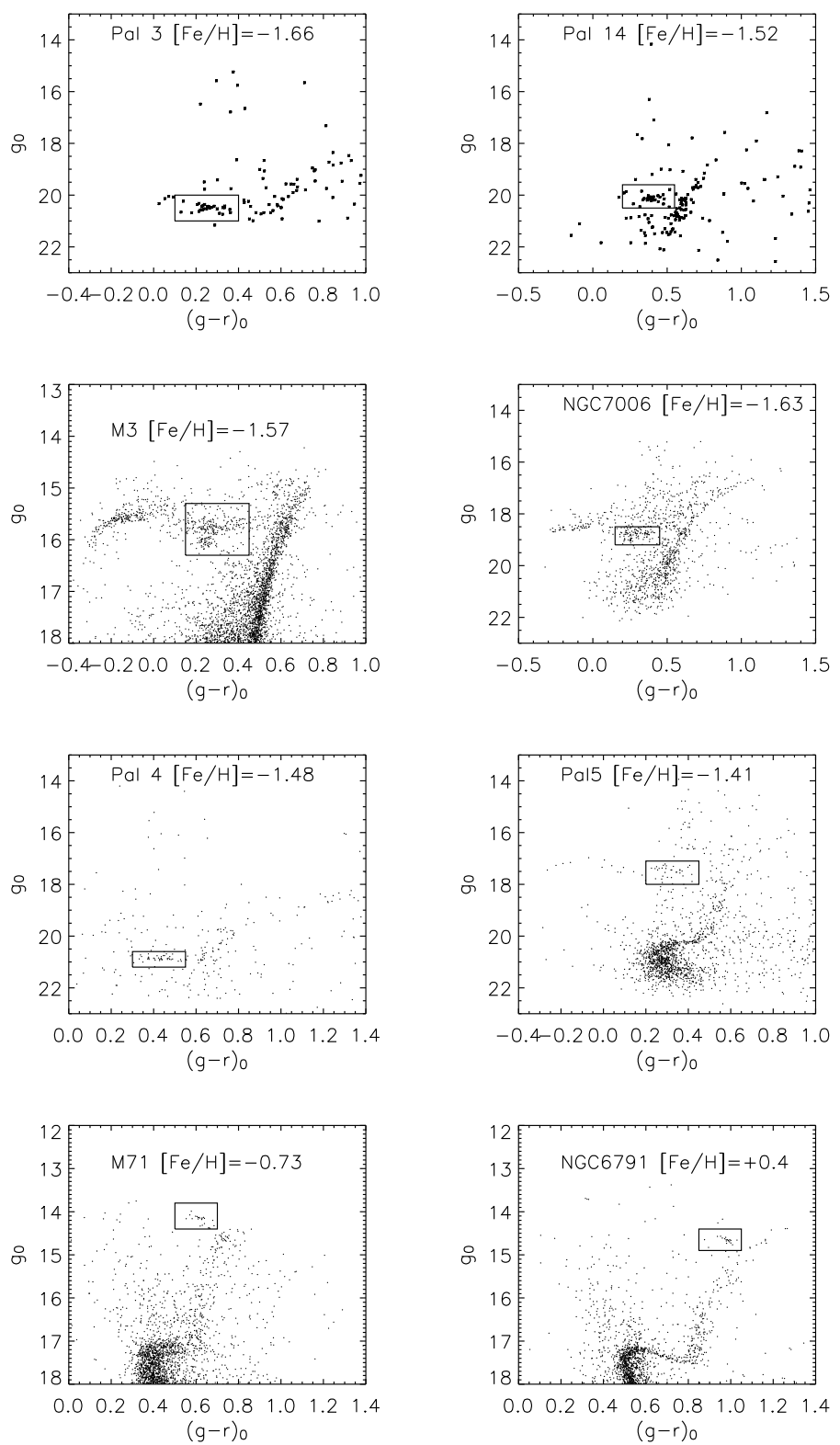

Fig. 3.- $(g-r)_{0}-g_{0}$ diagrams of the eight clusters. 
$g \sim 15 \mathrm{mag}$, which are significantly smaller than the broadening widths of 0.1-0.2 mag found in Figure 4.

With the distance modulus from Table 1 of An et al. (2008), ugriz magnitudes (after reddening correction) are translated into absolute magnitudes. Table 2 presented $(g-r)_{0}$ colors, absolute magnitudes and their scatters estimated from the widths of the Gaussian distributions for the clusters. It shows that $M_{\mathrm{u}}$ and $M_{\mathrm{g}}$ have quite large ranges and the deviations reach 2.7 and 1.0 mag respectively in the metallicity range of $-1.7 \leq[\mathrm{Fe} / \mathrm{H}] \leq$ +0.4 . The variations of $M_{\mathrm{r}}$ and $M_{\mathrm{z}}$ reduce to $0.35-0.45 \mathrm{mag}$ and $M_{\mathrm{i}}$ shows the smallest deviation of $0.16 \mathrm{mag}$ in this metallicity range. By using the relation of Chonis \& Gaskell (2008), it is possible to transfer $M_{\mathrm{g}}, M_{\mathrm{r}}$ and $M_{\mathrm{i}}$ into $M_{V}, M_{R}$, and $M_{I}$, and they are also presented in Table 2.

\subsection{Absolute Magnitudes as Functions of metallicity and age}

Absolute magnitudes in ugriz filters of RHB stars are investigated as functions of the cluster's metallicity and age in Figure 5. It shows that $M_{\mathrm{u}}$ has a good correlation with the cluster's metallicity and has only weak dependence on the cluster's age. $M_{\mathrm{g}}$ and $M_{\mathrm{r}}$ also increase with the increasing metallicity while $M_{\mathrm{i}}$ and $M_{\mathrm{z}}$ do not show significant variation in the metallicity range of $-1.7<[\mathrm{Fe} / \mathrm{H}]<+0.4$. For clusters with $[\mathrm{Fe} / \mathrm{H}]<-1.0, M_{\mathrm{u}}$ and $M_{\mathrm{g}}$ decrease, while $M_{\mathrm{i}}$ and $M_{\mathrm{z}}$ increase with increasing age, and $M_{\mathrm{r}}$ has little dependence on the cluster's age.

In order to estimate this sensitivity quantitatively, we perform multivariate fittings to the data in the formula $M x=a[\mathrm{Fe} / \mathrm{H}]+b t+c$, where $t$ indicates the cluster's age and $x=u, g, r, i, z, V, R$, and $I$. The coefficients and scatters of the calibrations are shown in Table 3. Note that the metallicities of globular clusters in the present work are taken from Harris (1996), Feb. 2003 version. They are found to agree well with those from Kraft \& Ivans (2003). As pointed out by An et al. (2009), the metalicities in Kraft \& Ivans (2003) are more reliable because they are derived from FeII lines, which usually do not suffer from NLTE effects and they agree well with those of Zinn \& West (1984). But, we note that the metallicity for M71 in Zinn \& West (1984) is too high (-0.58 vs. -0.81) as compared with that from Kraft \& Ivans (2003). The metallicity of M71 in Harris (1996), 2003 February version, is in between. We have checked that the results present in Table 3 will not be altered significantly when the metallicities from either Zinn \& West (1984) or Carretta \& Graton (1997) are used.

When we adopt the reddening and distance modulus from Grundahl et al. (2002) for 
Table 1: Critical radii $\left(R s\right.$, arcmin), $(g-r)_{0}$ color ranges and $g_{0}$ magnitude ranges of RHB stars, together with metallicities, ages, reddening and distance modulus of nine clusters.

\begin{tabular}{|c|c|c|c|c|c|c|c|}
\hline Cluster & $R s$ & $(g-r)_{0}$ range & $g_{0}$ range & {$[\mathrm{Fe} / \mathrm{H}]$} & Age & $E(B-V)$ & $(m-M)_{0}$ \\
\hline Pal3 & 4.56 & $0.10-0.40$ & $20.0-21.0$ & -1.66 & 9.7 & 0.04 & 19.84 \\
\hline NGC7006 & 4.23 & $0.15-0.45$ & $18.5-19.2$ & -1.63 & 11.0 & 0.05 & 18.09 \\
\hline M3 & 9.83 & $0.15-0.45$ & $15.3-16.3$ & -1.57 & 11.3 & 0.01 & 15.09 \\
\hline pal14 & 2.56 & $0.20-0.55$ & $19.6-20.5$ & -1.52 & 8.3 & 0.04 & 19.35 \\
\hline pal4 & 3.33 & $0.30-0.55$ & $20.6-21.2$ & -1.48 & 9.5 & 0.01 & 20.19 \\
\hline pal5 & 16.28 & $0.20-0.45$ & $17.1-18.0$ & -1.41 & 9.8 & 0.03 & 16.83 \\
\hline M5 & 28.40 & $0.05-0.45$ & $14.5-15.5$ & -1.27 & 10.9 & 0.03 & 14.37 \\
\hline M71 & 4.24 & $0.50-0.70$ & $13.8-14.4$ & -0.73 & 10.2 & 0.25 & 13.02 \\
\hline NGC6791 & 5.00 & $0.85-1.05$ & $14.5-15.5$ & 0.40 & 8.0 & 0.16 & 13.02 \\
\hline
\end{tabular}

Table 2: The centering colors and absolute magnitudes, together with their scatters, of RHB stars in nine clusters. The $M_{\mathrm{V}}, M_{\mathrm{R}}$ and $M_{\mathrm{I}}$ are also presented.

\begin{tabular}{rrrrrrrrrrrrrrrr}
\hline Cluster & $(g-r)_{0}$ & $\sigma$ & $M_{\mathrm{u}}$ & $\sigma M_{\mathrm{u}}$ & $M_{\mathrm{g}}$ & $\sigma M_{\mathrm{g}}$ & $M_{\mathrm{r}}$ & $\sigma M_{\mathrm{r}}$ & $M_{\mathrm{i}}$ & $\sigma M_{\mathrm{i}}$ & $M_{\mathrm{z}}$ & $\sigma M_{\mathrm{z}}$ & $M_{\mathrm{V}}$ & $M_{\mathrm{R}}$ & $M_{\mathrm{I}}$ \\
\hline Pal3 & 0.25 & 0.08 & 1.71 & 0.25 & 0.60 & 0.13 & 0.28 & 0.08 & 0.28 & 0.08 & 0.28 & 0.10 & 0.40 & 0.12 & -0.09 \\
NGC7006 & 0.31 & 0.13 & 1.80 & 0.15 & 0.60 & 0.30 & 0.37 & 0.22 & 0.27 & 0.26 & 0.27 & 0.26 & 0.45 & 0.18 & -0.13 \\
M3 & 0.28 & 0.12 & 1.80 & 0.22 & 0.64 & 0.21 & 0.40 & 0.30 & 0.31 & 0.30 & 0.31 & 0.32 & 0.49 & 0.22 & -0.09 \\
pal14 & 0.40 & 0.12 & 1.91 & 0.27 & 0.77 & 0.06 & 0.35 & 0.15 & 0.15 & 0.16 & 0.07 & 0.22 & 0.51 & 0.14 & -0.28 \\
pal4 & 0.40 & 0.09 & 1.88 & 0.35 & 0.60 & 0.13 & 0.20 & 0.08 & 0.17 & 0.08 & -0.05 & 0.15 & 0.36 & 0.04 & -0.21 \\
pal5 & 0.29 & 0.08 & 1.71 & 0.07 & 0.62 & 0.24 & 0.25 & 0.19 & 0.25 & 0.19 & 0.14 & 0.27 & 0.39 & 0.09 & -0.12 \\
M5 & 0.27 & 0.15 & 1.84 & 0.14 & 0.67 & 0.17 & 0.39 & 0.17 & 0.30 & 0.20 & 0.30 & 0.23 & 0.50 & 0.21 & -0.10 \\
M71 & 0.60 & 0.02 & 2.67 & 0.08 & 1.09 & 0.04 & 0.48 & 0.03 & 0.23 & 0.04 & 0.16 & 0.04 & 0.72 & 0.25 & -0.22 \\
NGC6791 & 0.98 & 0.03 & 4.42 & 0.27 & 1.60 & 0.07 & 0.65 & 0.06 & 0.31 & 0.05 & 0.15 & 0.07 & 1.03 & 0.40 & -0.17 \\
\hline \multicolumn{1}{c}{ New reddening } & and distance modulus suggested by An et al. 2009$)$ & & \\
\hline M71 & 0.62 & 0.09 & 2.71 & 0.07 & 1.20 & 0.15 & 0.64 & 0.15 & 0.36 & 0.11 & 0.27 & 0.07 & 0.86 & 0.40 & -0.11 \\
NGC6791 & 1.04 & 0.02 & 4.65 & 0.26 & 1.82 & 0.07 & 0.80 & 0.06 & 0.44 & 0.08 & 0.24 & 0.06 & 1.21 & 0.54 & -0.06 \\
\hline
\end{tabular}



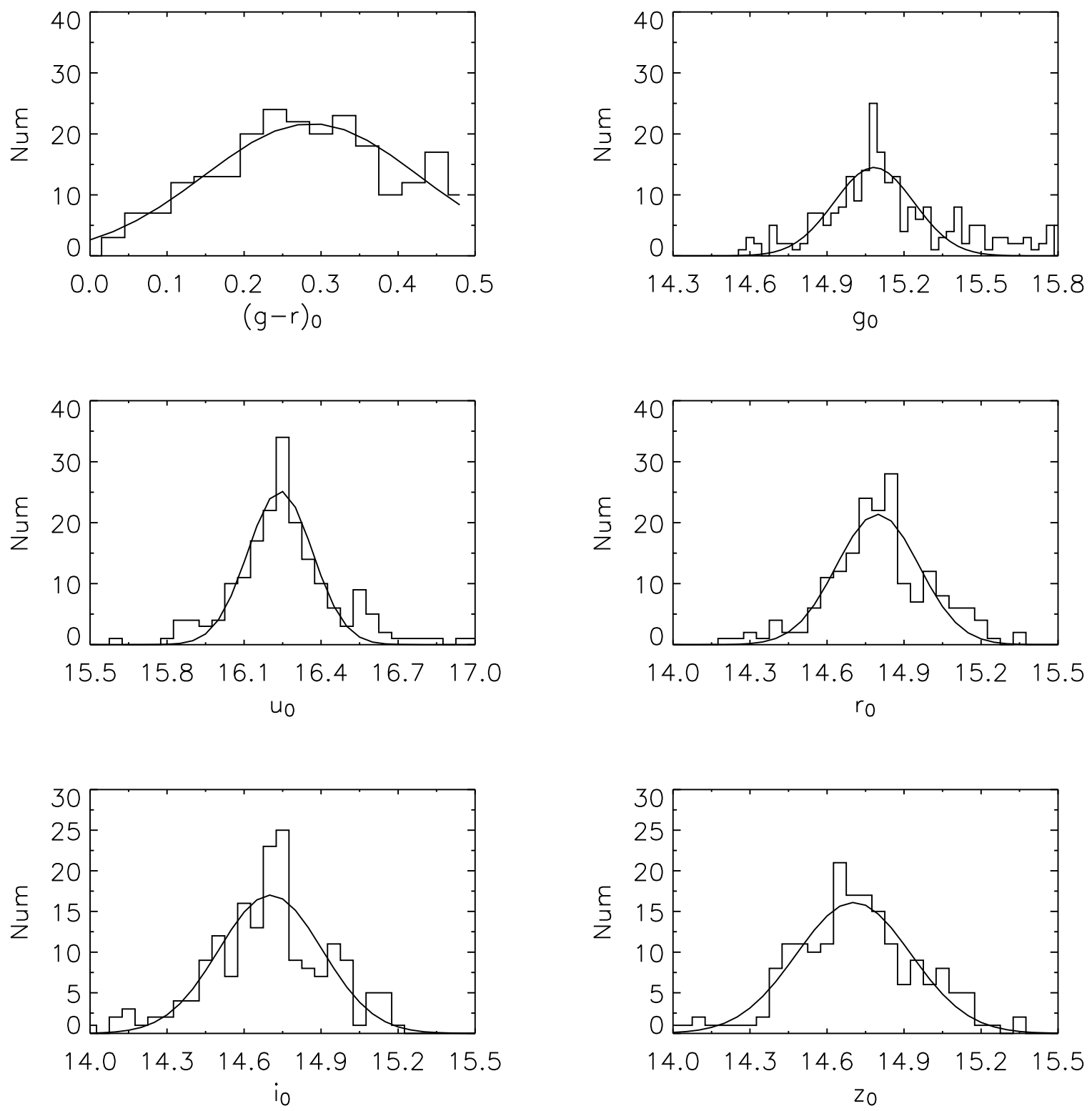

Fig. 4.- The $(g-r)_{0}$ color and ugriz magnitude distributions, together with their Gaussian fittings, of the selected RHB stars for M5. 

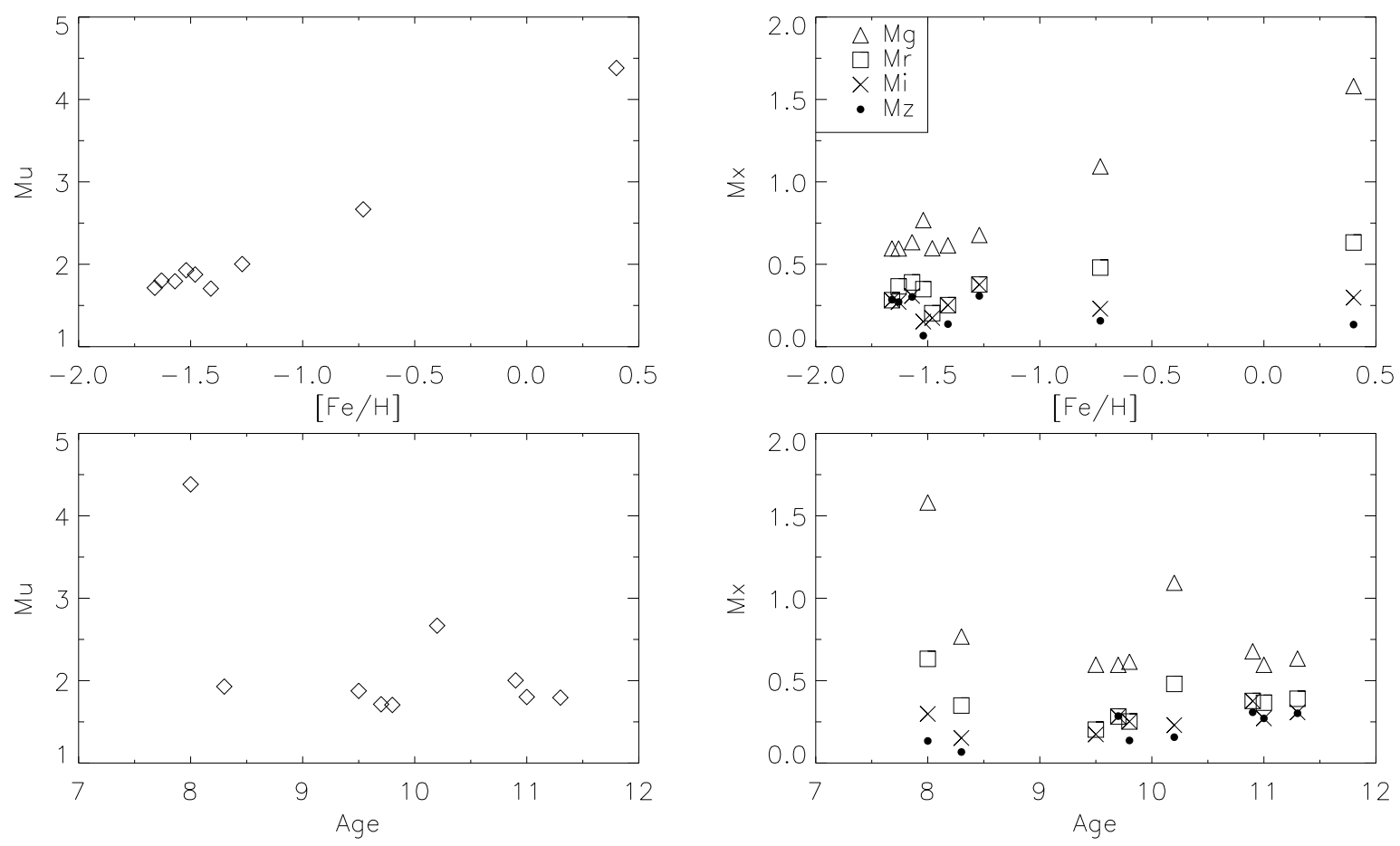

Fig. 5.- $M_{\mathrm{X}},(x=u, g, r, i$, and $z$ indicated by diamonds, triangles, squares, crosses and circles respectively) vs. $[\mathrm{Fe} / \mathrm{H}]$ and age.

Table 3: The coefficients, $a, b, c$, and their errors as well as the scatters of the calibrations in the formula of $M=a[\mathrm{Fe} / \mathrm{H}]+b t+c$. The reddening and distance modulus are taken from Harris (1996) 2003 version for globular clusters and from An et al. (2008) for NGC6791.

\begin{tabular}{rrrrrrr}
\hline Filters & $a$ & $b$ & $c$ & $\sigma_{a}$ & $\sigma_{b}$ & $\sigma$ \\
$M_{\mathrm{u}}$ & 1.243 & -0.046 & 4.158 & 0.095 & 0.055 & 0.132 \\
$M_{\mathrm{g}}$ & 0.469 & -0.026 & 1.614 & 0.047 & 0.027 & 0.065 \\
$M_{\mathrm{r}}$ & 0.192 & 0.030 & 0.311 & 0.043 & 0.025 & 0.060 \\
$M_{\mathrm{i}}$ & 0.064 & 0.049 & -0.148 & 0.034 & 0.020 & 0.047 \\
$M_{\mathrm{z}}$ & 0.041 & 0.082 & -0.576 & 0.065 & 0.037 & 0.090 \\
$M_{\mathrm{V}}$ & 0.306 & 0.007 & 0.838 & 0.043 & 0.025 & 0.060 \\
$M_{\mathrm{R}}$ & 0.157 & 0.035 & 0.025 & 0.033 & 0.019 & 0.046 \\
$M_{\mathrm{I}}$ & 0.021 & 0.056 & -0.671 & 0.047 & 0.027 & 0.065 \\
\hline
\end{tabular}


M71, the absolute magnitudes deviate by 0.04-0.16 mag. For NGC6791, we adopt E(B$\mathrm{V})=0.16$ and $(m-M)_{0}=13.02$, which agree with the results from Carney et al. (2005: 0.14/13.07) based on JHK photometry, Anthony-Twarog et al. (2007: 0.16/13.10) based on uvby photometry and Bedin et al. (2008: 0.17/12.97) based on HST/ACS photometry. When the new values (0.10/13.02) of NGC6791 from An et al. (2009) are adopted, the colors and absolute magnitudes change significantly. The coefficients in the calibrations are presented in Table 4. It shows that dependence of absolute magnitude on metallicity in the calibrations are increased significantly. For example, metallicity dependence in the $M_{\mathrm{V}}$ calibration is as high as 0.41 , which is not previously found in any calibration for RR Lyrae stars and RC stars. For this reason, we do not adopt the results based on new reddening in Table 4, which is presented in this paper for reference.

A few interesting results can be drawn from Table 3. First, it shows that $M_{\mathrm{i}}$ has the smallest dependence on metallicity and age as well as the smallest scatter. Second, the relations for $V R I$ filters are quite similar to those for gri filters, but they show less dependence on metallicity. Finally, the most interesting result is that dependence of absolute magnitude on metallicity and age shows different directions. The metallicity coefficient decreases, while the age coefficient increases, as the filter varies from blue $u$ to red $z$ in the formula of $M=a[\mathrm{Fe} / \mathrm{H}]+b t+c$. The $b$ coefficient increases when coefficient $a$ decreases; they show a nonlinear relation as shown in Figure 6. Meanwhile, the constant $c$ decreases with decreasing $a$ coefficient, and they show a linear trend.

\subsection{Traditional calibrations with metallicity}

In order to investigate the age effect on the above calibrations, we carry out the regressions of absolute magnitude with metallicity in the traditional formula of $M=a[\mathrm{Fe} / \mathrm{H}]+c$. The coefficients $a$ and the constants $c$ obtained from the linear fittings to the data are presented in Table 5. The results based on nonlinear calibrations in the formula of $M=$ $a[\mathrm{Fe} / \mathrm{H}]+b[\mathrm{Fe} / \mathrm{H}]^{2}+c$ are given in Table 6.

It shows that $M_{\mathrm{u}}$ calibrations are slightly improved with smaller scatters when the formula includes the metallicity only, and the nonlinear calibration with metallicity is the best to reduce the scatter from 0.14 to $0.09 \mathrm{mag}$. For the other four griz filters, nonlinear

calibrations with metallicity are not necessary because they give exactly the same scatters and the same constant $c$. As compared with the calibrations with the age term included in Sect. 3.2, it shows that age plays a minor role to reduce the scatters and the metallicity coefficient $a$ does not change too much while the constant $c$ deviates from 0.2 to 0.7 mag as shown in Figure6. 
Table 4: The coefficients, $a, b, c$, and the scatters of the calibrations in the formula of $M=a[\mathrm{Fe} / \mathrm{H}]+b t+c$. The reddening and distance modulus of M71 are taken from Grundahl et al. (2002) and the reddening from An et al. (2009) is used for NGC6791.

\begin{tabular}{rrrrrrr}
\hline filters & $a$ & $b$ & $c$ & $\sigma_{a}$ & $\sigma_{b}$ & $\sigma$ \\
$M_{\mathrm{u}}$ & 1.361 & -0.055 & 4.428 & 0.109 & 0.063 & 0.151 \\
$M_{\mathrm{g}}$ & 0.590 & --0.026 & 1.807 & 0.055 & 0.032 & 0.076 \\
$M_{\mathrm{r}}$ & 0.295 & 0.037 & 0.394 & 0.052 & 0.030 & 0.072 \\
$M_{\mathrm{i}}$ & 0.148 & 0.055 & -0.074 & 0.026 & 0.015 & 0.036 \\
$M_{\mathrm{z}}$ & 0.112 & 0.088 & -0.524 & 0.063 & 0.037 & 0.088 \\
$M_{\mathrm{V}}$ & 0.417 & 0.011 & 0.967 & 0.053 & 0.031 & 0.073 \\
$M_{\mathrm{R}}$ & 0.255 & 0.042 & 0.106 & 0.039 & 0.023 & 0.054 \\
$M_{\mathrm{I}}$ & 0.098 & 0.061 & -0.601 & 0.037 & 0.022 & 0.052 \\
\hline
\end{tabular}

Table 5: The coefficients, errors and scatters in the $M=a[\mathrm{Fe} / \mathrm{H}]+c$ formula.

\begin{tabular}{crrrr}
\hline Filters & $a$ & $c$ & $\sigma_{a}$ & $\sigma$ \\
$M_{\mathrm{u}}$ & 1.284 & 3.759 & 0.079 & 0.139 \\
$M_{\mathrm{g}}$ & 0.492 & 1.390 & 0.040 & 0.070 \\
$M_{\mathrm{r}}$ & 0.165 & 0.569 & 0.038 & 0.066 \\
$M_{\mathrm{i}}$ & 0.019 & 0.283 & 0.038 & 0.068 \\
$M_{\mathrm{z}}$ & -0.033 & 0.139 & 0.068 & 0.120 \\
$M_{\mathrm{V}}$ & 0.300 & 0.897 & 0.034 & 0.060 \\
$M_{\mathrm{R}}$ & 0.125 & 0.332 & 0.033 & 0.058 \\
$M_{\mathrm{I}}$ & -0.030 & -0.183 & 0.048 & 0.085 \\
\hline
\end{tabular}

Table 6: The coefficients, errors and scatters in the formula of $M=a[\mathrm{Fe} / \mathrm{H}]+b[\mathrm{Fe} / \mathrm{H}]^{2}+c$.

\begin{tabular}{rrrrrrr}
\hline Filters & $a$ & $b$ & $c$ & $\sigma_{a}$ & $\sigma_{b}$ & $\sigma$ \\
$M_{\mathrm{u}}$ & 1.688 & 0.313 & 3.667 & 0.144 & 0.103 & 0.087 \\
$M_{\mathrm{g}}$ & 0.507 & 0.011 & 1.387 & 0.115 & 0.083 & 0.070 \\
$M_{\mathrm{r}}$ & 0.178 & 0.010 & 0.566 & 0.109 & 0.078 & 0.066 \\
$M_{\mathrm{i}}$ & 0.034 & 0.012 & 0.279 & 0.111 & 0.080 & 0.068 \\
$M_{\mathrm{z}}$ & 0.014 & 0.037 & 0.129 & 0.197 & 0.142 & 0.120 \\
$M_{\mathrm{V}}$ & 0.314 & 0.011 & 0.894 & 0.098 & 0.071 & 0.060 \\
$M_{\mathrm{R}}$ & 0.138 & 0.010 & 0.329 & 0.095 & 0.068 & 0.058 \\
$M_{\mathrm{I}}$ & -0.014 & 0.013 & -0.187 & 0.139 & 0.100 & 0.085 \\
\hline
\end{tabular}



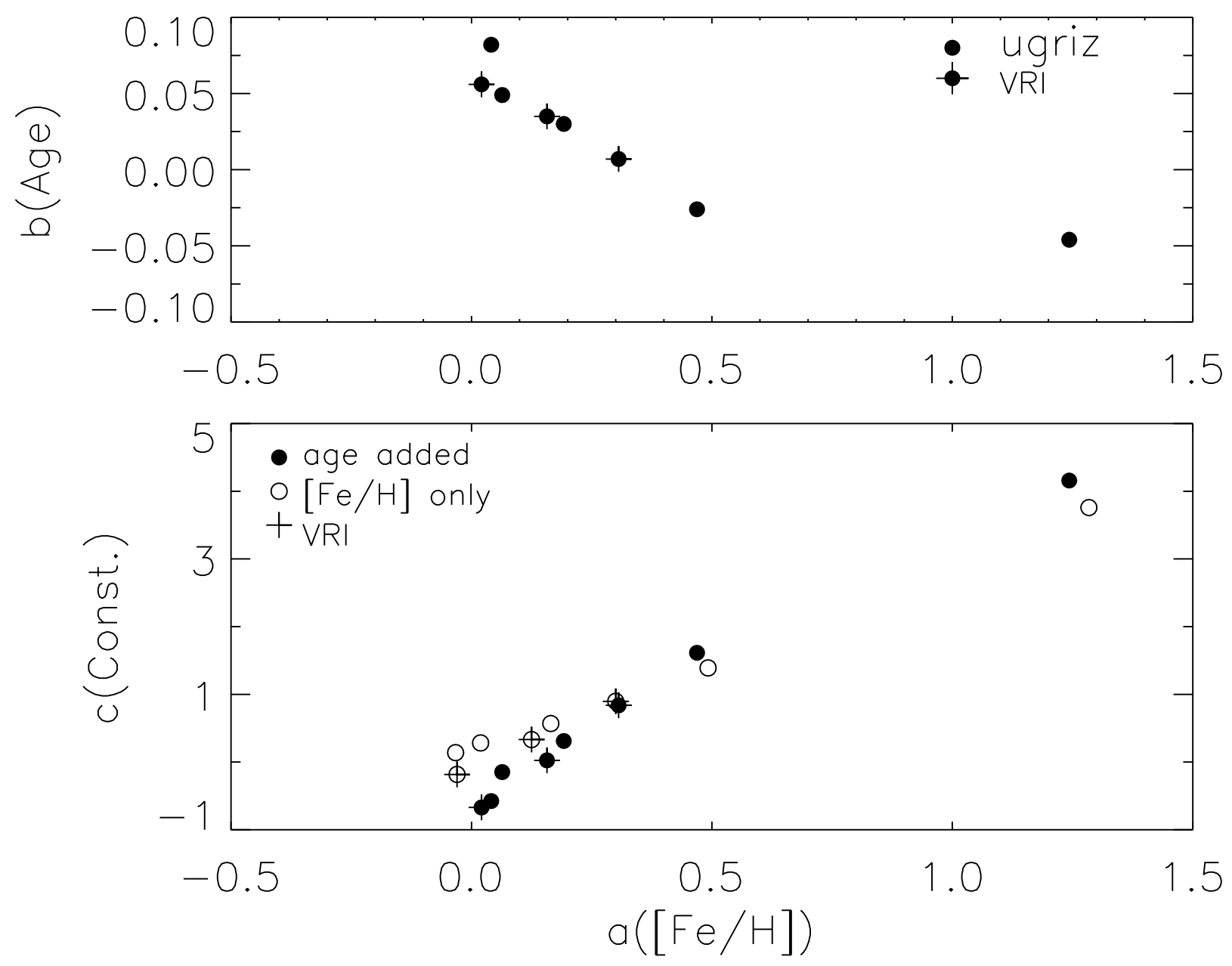

Fig. 6. - Trends of age coefficient $b$ and constant $c$ vs. the metallicity coefficient $a$ in the formula of $M=a[\mathrm{Fe} / \mathrm{H}]+b t+c$ (filled circles) and $M=a[\mathrm{Fe} / \mathrm{H}]+c$ (open circles). The additional plus indicates data based on $R V I$ filters. 
In the comparison of the calibrations between narrow band gri and wide band $V R I$ filters, it shows that the metallicity coefficient reduces as the wide band is adopted, which does not indicate less dependence on metallicity. As shown in the case of $M_{\mathrm{i}}$ and $M_{\mathrm{I}}$ calibrations in the the traditional formulae of $M=a[\mathrm{Fe} / \mathrm{H}]+c$, the $a$ coefficient reduces from 0.02 in the $M_{\mathrm{i}}$ calibration to -0.03 in the $M_{\mathrm{I}}$ calibration, but the dependence on metallicity in the $M_{\mathrm{i}}$ calibration is smaller than that in the $M_{\mathrm{I}}$ calibration. When the age effect is included in the calibrations, it shows that the metallicity dependence in the wide band $V R I$ calibrations is smaller than that in narrow band gri filters. But the age coefficient and the constant in these calibrations change accordingly. In fact, both the metallicity and age dependence have a minimum at a particular wavelength band. The minimum metallicity dependence is found at a band redder than $I$ and the minimum age dependence lies between $g$ and $r$ in the $M=a[\mathrm{Fe} / \mathrm{H}]+b t+c$ calibrations.

\subsection{The comparison of absolute magnitudes from different stars}

$M_{\mathrm{g}}$ values of RHB at the metal poor edge are about 0.6-0.7 mag for an old population with an age larger than $10 \mathrm{Gyr}$. These values are quite consistent with theoretical $M_{\mathrm{g}}$ values of BHB as shown in Figure 8 of Sirko et al. (2004) where $M_{\mathrm{g}}$ values of BHB are 0.6-0.7 mag for $(u-g)_{0}=1.1-1.2 \mathrm{mag}$. Note that $(u-g)_{0}$ values of our RHB are also in the same range of 1.1-1.2 mag for the metal poor and old clusters, while $(g-r)_{0}$ values are about 0.3-0.4 mag for RHB versus $-0.2 \mathrm{mag}$ for BHB at $(u-g)_{0}=1.1-1.2 \mathrm{mag}$. However, we notice that $M_{\mathrm{g}}$ of RHB at $(g-r)_{0}=0.3 \mathrm{mag}$ should be slightly fainter than that of BHB at $(g-r)_{0}=-0.2$ by $0.2 \mathrm{mag}$ as we inspect the $(g-r)_{0^{-}} g_{0}$ diagrams of M5 (in Figure2), M3 and NGC 7006 (in Fig 3). Moreover, we notice that $g_{0}$ of BHB in M5 decreases by 0.4 mag as the $(g-r)_{0}$ values vary from 0.0 to $-0.2 \mathrm{mag}$ and by $1.5 \mathrm{mag}$ as the $(g-r)_{0}$ values vary from 0.0 to $-0.5 \mathrm{mag}$, which is the color range that Sirko et al. (2004) select BHB stars. But, the average $M_{\mathrm{g}}$ of BHB give the same values of our RHB. This may explain the consistent $M_{\mathrm{g}}$ values of our RHB with theoretical values of BHB presented in Sirko et al. (2004). However, it is clear that $M_{\mathrm{g}}$ of BHB do vary with $(g-r)_{0}$ colors. In this sense, absolute magnitudes of BHB stars should be determined by taking into account their color variations.

In comparison with the calibration of $M_{\mathrm{V}}=0.30[\mathrm{Fe} / \mathrm{H}]+0.92$ by McNamara (2001) based on RR Lyrae stars in clusters, deviations of $M_{\mathrm{V}}(\mathrm{RHB}-\mathrm{HB})$ are about $0.02 \mathrm{mag}$ for clusters in group 1. To some extent, these agreements may indicate that both $M_{\mathrm{g}}$ of RHB stars derived in the present work and the translation relations of Chonis \& Gaskell (2008) are quite reliable. The deviations slightly increase to $0.07-0.15 \mathrm{mag}$ for clusters in group 2 due to 
their young ages. Since the calibration by McNamara (2001) does not include the age term and it is based on an old population, it may not be valid for young populations. In this sense, new calibrations should be established for metallicity higher than $[\mathrm{Fe} / \mathrm{H}] \sim-1.5$, where more stars are younger in the context of Galactic evolution. In other words, the age term is important in establishing these calibrations in the metallicity range of $-1.5<[\mathrm{Fe} / \mathrm{H}]<-0.5$.

For a metal rich population, $\mathrm{RC}$ stars are good distance indicators. Recent work by Groenewegen (2008) gives the calibration of $M_{\mathrm{I}}=0.08([\mathrm{Fe} / \mathrm{H}]+0.15)-0.26$ based on the revised Hipparcos parallaxes by van Leeuwen (2007). The deviation of $M_{\mathrm{I}}$ (RHB-RC) is 0.03 mag for NGC6791 at $[\mathrm{Fe} / \mathrm{H}]=+0.4$ and $0.07 \mathrm{mag}$ for $\mathrm{M} 71$ at $[\mathrm{Fe} / \mathrm{H}]=-0.7$. Extending the relation of Groenewegen (2008) to low metallicity of $[\mathrm{Fe} / \mathrm{H}]=-1.5$, the deviations of $M_{\mathrm{I}}$ (RHB-RC) increase from 0.08 to $0.28 \mathrm{mag}$ when age increases from 8.3 to 11.3 Gyr. The deviations of $M_{\mathrm{V}}(\mathrm{RHB}-\mathrm{HB})$ and $M_{\mathrm{I}}(\mathrm{RHB}-\mathrm{RC})$ are shown in Figure 7 , where clusters in group 1 (open) and group 2 (filled) are shown separately. Again, the deviations are mainly introduced due to age differences between RC stars and globular clusters because $M_{\mathrm{I}}$ calibrations are quite sensitive to age. Without the age term, $M_{\mathrm{I}}$ calibrations cannot be established well as shown in Section 3.3, where the uncertainty of metallicity coefficient and the scatter in the $M_{\mathrm{I}}$ calibration become quite large. It seems that absolute magnitudes of BHB, RHB and RC are consistent when both metallicity and age are taken into account.

\section{Implications and Conclusions}

Using the $(g-r)_{0}-g_{0}$ diagrams of stars in the central parts of clusters based on the SDSS survey from An et al. (2008), we have selected RHB stars in nine clusters covering the metallicity of $1.7<[\mathrm{Fe} / \mathrm{H}]<+0.4$ and the age of 8-12 Gyr. Absolute magnitudes of RHB stars in the SDSS ugriz system are derived and they are translated into the Johnson $V R I$ system based on the relations of Chonis \& Gaskell (2008). The calibrations of $M_{\mathrm{X}}$ (where $X=u, g, r, i, z, V, R, I$ ) with metallicity and age are established and they can be used to study the thick disk population which has the same metallicity and age ranges as these clusters.

Based on an analysis of these data, we have found that $M_{\mathrm{i}}=0.06[\mathrm{Fe} / \mathrm{H}]+0.040 t+0.03$ is the calibration for distance determination due to its smallest scatter of 0.04 mag when both metallicity and age are known. On the other hand, it is also the best filter for distance determination when both metallicity and age are unknown because it has the least sensitivity to metallicity and age. The scatters in $M_{\mathrm{g}}$ and $M_{\mathrm{r}}$ calibrations are also small (0.06 mag) but their sensitivities to metallicity become larger. They can be used for distance determination if the metallicity is known and the metallicity range of the population is narrow. For a 

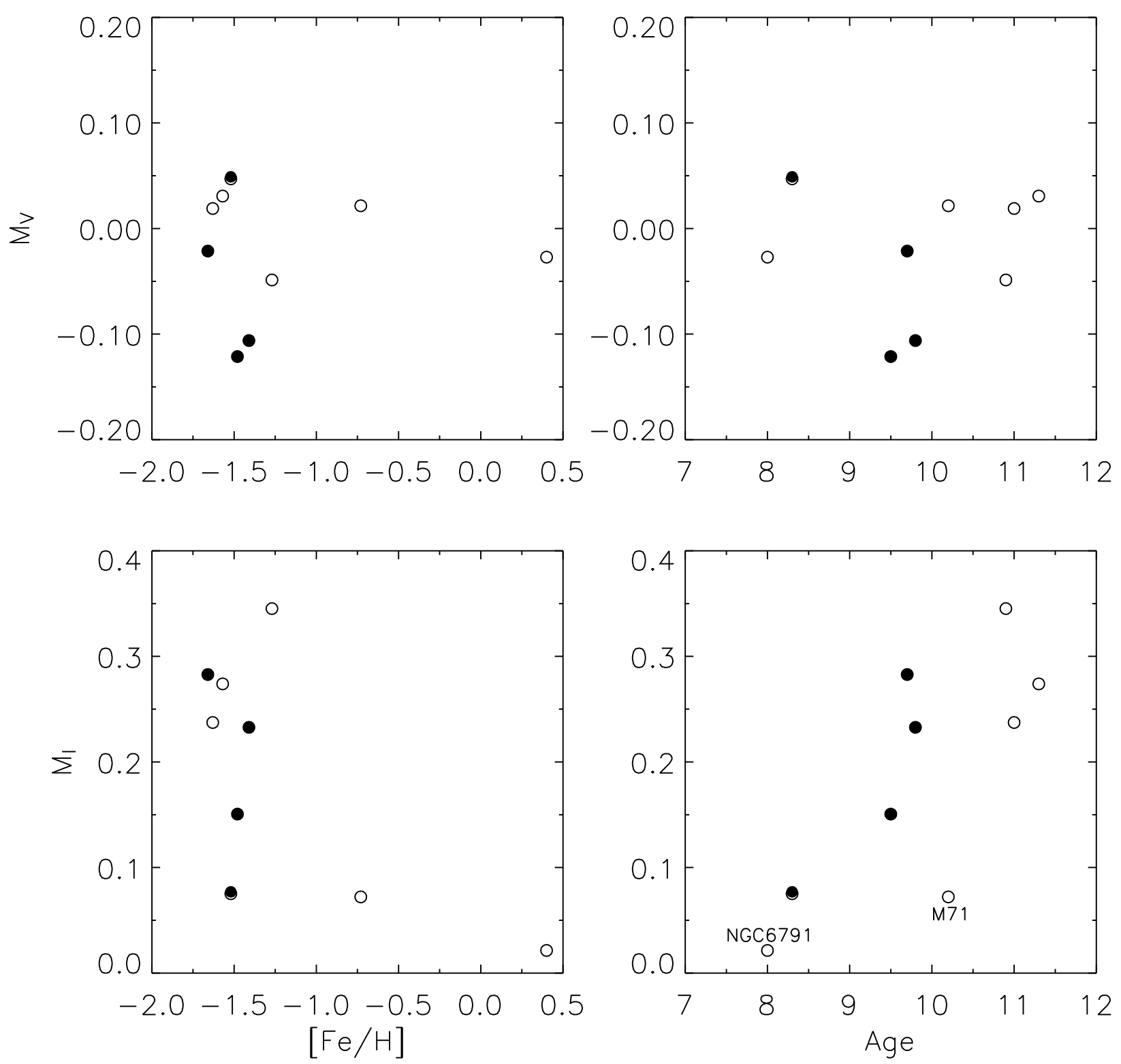

Fig. 7.- $M_{\mathrm{V}}$ and $M_{\mathrm{I}}$ of RHB stars for clusters in group 1 (filled) and 2 (open) are compared with those of $\mathrm{BHB}$ and $\mathrm{RC}$ stars in the literature. 
population with known metallicity but large age span, the calibration in the $V$ filter may be better due to its smaller dependence on age. For a population with both large metallicity and large span in age, e.g. RC stars in the thin disk, the widely adopted $M_{\text {I }}$ filter may not be the best choice. The deviations could be as large as $0.5 \mathrm{mag}$ in $M_{\mathrm{I}}$ versus $0.25 \mathrm{mag}$ in $M_{\mathrm{V}}$ when metallicity varies from -0.5 to 0.0 and age varies from 0 to $8 \mathrm{Gyr}$, which is typical for stars in the thin disk.

In particular, we have found that coefficients of metallicity and age in our calibrations show the opposite trends. This provides a challenge to the previous suggestion that the calibration of absolute magnitude in $I$ filter is better than that in the $V$ filter. Without the age term included in the calibration, it is difficult to decide which is the best one. In the present work, $M_{\mathrm{g}}$ and $M_{\mathrm{z}}$ show significant and opposite dependence on age. For $[\mathrm{Fe} / \mathrm{H}]=-1.5$, an age difference of 3 Gyr introduces deviations of $0.2-0.4 \mathrm{mag}$ in $M_{\mathrm{g}}$ and $M_{\mathrm{z}}$. Also, we provide the evidence on age dependence of the $M_{\mathrm{I}}$ calibration, which should be investigated in the future.

We thank the anonymous referee for his valuable suggestions towards improving the paper. Dr. James Wicker is thanked for careful inspection on the written English of this paper. This study is supported by the National Natural Science Foundation of China under grants 10821061, 10673015, the National Basic Research Program of China (973 program) No. 2007CB815103/815403, and by the Ministry of Science and technology of China under grant 2006AA01A120.

\section{REFERENCES}

An, D., et al. 2008, ApJS, 179, 326

An, D., et al. 2009, ApJ, 700, 723

Anthony-Twarog, B.J., Twarog, B.A., \& Mayer, L. 2007, 133, 1535

Bedin, L.R., et al. 2008, ApJ, 678, 1279

Carney, B.W., Lee, J.W. \& Dodson, B. 2005, AJ 129, 656

Carretta, E., Gratton, R.G., Clementini, G., Fusi Pecci, F. 2000, ApJ, 533, 215

Carretta, E., \& Gratton, R. 1997, A\&AS, 121, 95

Chonis, T.S. \&, Gaskell, C.M., 2008, AJ, 135, 264 
Feast, M.F 1997, MNRAS, 284, 76

Groenewegen, M.A.T. 2008, A\&A, 488, 935

Grundahl, F., Stetson, P.B., \& Andersen, M.I. 2002, A\&A, 395, 481

Harris, W.E. 1996, AJ, 112, 1487

Kraft R.P. \& Ivans I.I., 2003, PASP, 115, 143

McNamara, D.H. 2001, PASP, 113,335

Salaris, M \& Weiss, W. 2002, A\&A, 388, 492

Sandage, A. \& Tammann, G.A. 2006, ARA\&A, 44, 93

Sirko, E., et al. 2004, AJ, 127, 899

Stanek, K.Z. \& Garnavich, P.M. 1998, ApJ, 503, L131

van Leeuwen, F. 2007, A\&A, 474, 653

van den Bergh, S. 2006, Astron. J. 131, 1559

Zhao, G., Qiu, H., Mao, S.D. 2001, ApJ, 551, L85

Zinn, R., \& West, M.J. 1984, ApJS, 55, 45 